\title{
Design of a flexibly-constrained revolute pair with non-linear stiffness in multiple directions
}

\author{
Naoto KIMURA*, Nobuyuki IWATSUKI ${ }^{* *}$ and Ikuma IKEDA** \\ * Graduate student, Department of Mechanical Engineering, Tokyo Institute of Technology \\ 2-12-1 O-okayama, Meguro-ku, Tokyo, 152-8552, Japan \\ E-mail: kimura.n.ac@m.titech.ac.jp \\ ** Department of Mechanical Engineering, Tokyo Institute of Technology \\ 2-12-1 O-okayama, Meguro-ku, Tokyo, 152-8552, Japan
}

Received: 20 January 2020; Revised: 27 April 2020; Accepted: 9 June 2020

\begin{abstract}
In order to synthesize a human-friendly flexible mechanism with a simple structure, a revolute pair with flexible kinematic constraint in multiple directions is proposed. It is called the multi-directionally flexibly constrained revolute pair (MFCRP). The structure of the MFCRP is composed of a link with two same spherical surfaces and a link with two same cam surfaces, and each set of the cam surface and the spherical surface is in contact at a point. The connection between the two links is kept by two linear springs arranged between the two links. The MFCRP can generate 1-axial relative rotation between the two links and relative motions in the other directions are flexibly constrained. In order for the MFCRP to have both flexibility for safety and rigidity for the force transmission, the specified non-linear stiffness can be implemented in the two relative translational directions between the two links. This flexible translational constraint is generated by the spring forces and the reaction forces between the two links. In this paper, two methods to design the cam surfaces to implement the specified non-linear stiffness are proposed. The validity of the proposed design methodology is confirmed by comparing measured stiffness characteristics between two links of some prototypes with the theoretical characteristics. As an application, a flexible closed-loop linkage with the MFCRP is fabricated and its flexibility and kinematic performance are investigated through some experiments.
\end{abstract}

Keywords : Linkage, Kinematic pair, Higher pair, Non-linear stiffness, Passive compliance, Cam profile design, Underactuated mechanism

\section{Introduction}

Flexible mechanisms are required for machines used in human daily life in order not to injure the users. However, flexibility for safety and rigidity for force transmission in the mechanism are generally in a trade-off relationship. Therefore, it is important to balance them.

Flexible mechanisms can be achieved as an underactuated mechanism whose redundant mobility is constrained with elastic elements. In order for it to have both flexibility and rigidity, many approaches to control compliance around rotational axes of its revolute pairs have been proposed. One of the approaches is the active compliance approach (Salisbury, 1980) (Hogan, 1991). This approach can control the compliance of an active revolute pair by controlling the impedance of a rotary actuator, such as a motor. However, a sophisticated control system is required to respond to a sudden change of an external force, such as dynamic collision. Another approach is the passive compliance approach. In this approach, rotational passive compliance is implemented in a revolute pair with some elastic elements, such as linear springs. In order for it to have both flexibility and rigidity, methods to adjust the passive compliance to the desired compliance with an additional actuator have been proposed by several researchers. These methods can be achieved by antagonizing non-linear springs (Migliore et al., 2005) (Palli et al., 2011) (Petit et al., 2015), changing the transmission of spring forces (Kim and Song, 2010) (Jafali et al., 2014), or changing preload applied to the springs (Wolf and Hirzinger, 2008) (Wolf et al., 2011) 


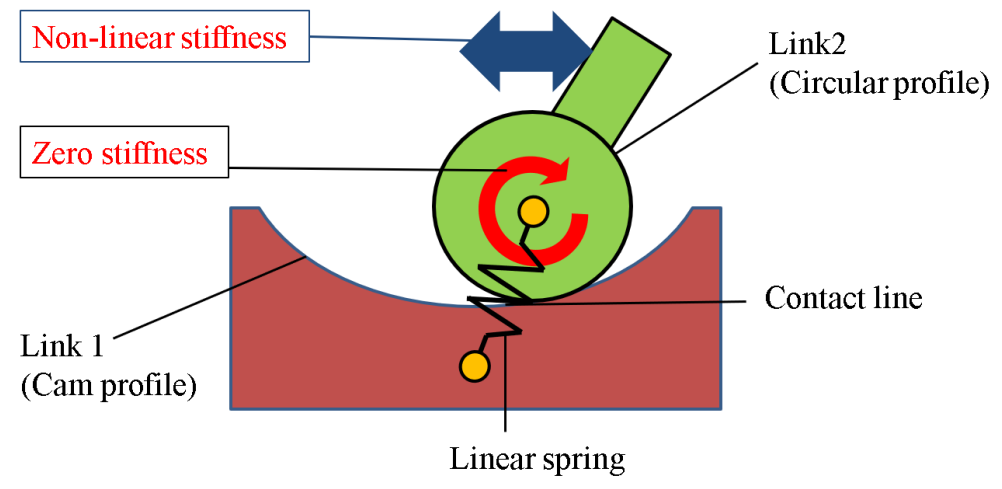

Fig. 1 The concept of the flexibly constrained revolute pair (FCRP) (Kimura et al., 2019)

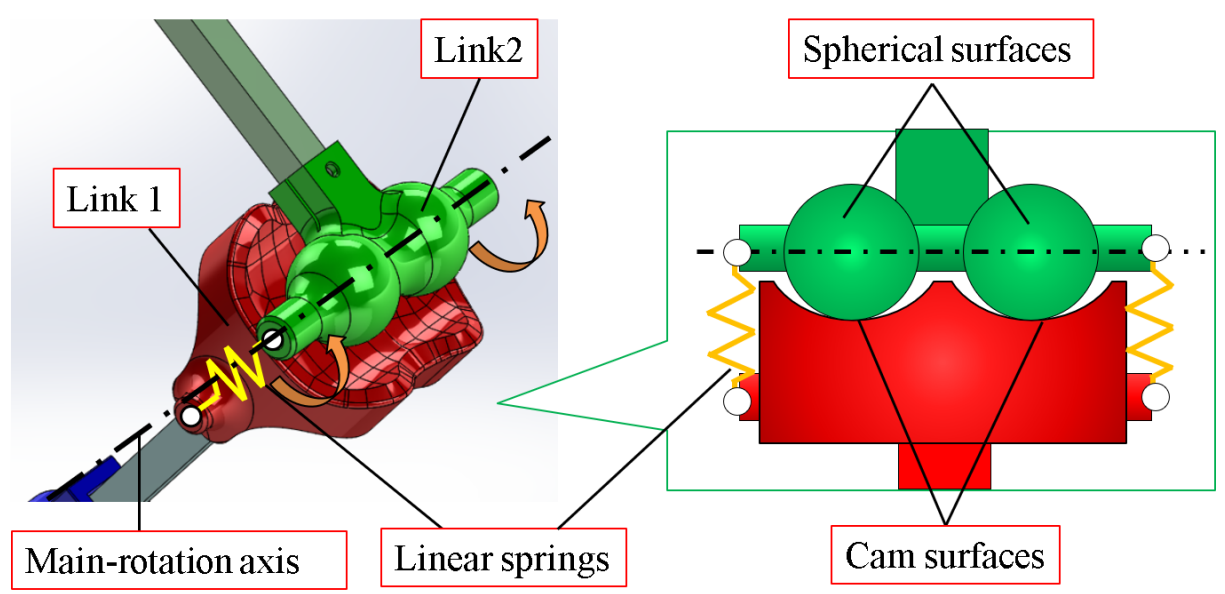

Fig. 2 The structure of the multi-directionally FCRP (MFCRP)

with an additional actuator. However, those mechanisms tend to be heavy because of the additional actuator. As another approach, the passive compliance approach with a non-linear stiffness has been proposed by several researchers. Joint mechanisms with this method tend to be lightweight because an additional actuator is not required. Non-linear stiffness has both flexibility and rigidity because it has both low and high stiffness area. For example, hardening stiffness characteristics can make a braking time in the small stiffness area and suppress large displacement in the large stiffness area when an external load is applied. Thus, it is suitable for applications requiring low precision and a large payload. Revolute pairs with non-linear rotational stiffness have been achieved by changing the displacement of elastic elements with a linkage (Okada and Kino, 2008) (Park and Song, 2010), cam mechanisms (Bidgory et al., 2016) (Kashiri, et al., 2017) or non-circular pulleys (Schepelmann et al., 2014) (Kim and Deshpande, 2014). However, those joint mechanisms are not so simple because of the large number of mechanical elements. In order to simplify the structure of a flexible revolute pair, the authors have proposed the flexibly constrained revolute pair (FCRP) (Kimura et al., 2019), which is a passive revolute pair with non-linear stiffness in a relative translational direction in the motion plane. The schematic diagram of the FCRP is shown in Fig.1. It has a simple structure composed of only two cylindrical cams kept in contact at a line and two linear springs connecting the two pairing elements. If the two links keep in contact at the line, this kinematic pair allows two degree of freedom (DOF) composed of a relative rotation around the center axis of the circular cylinder and a translation in the motion plane. In the relative translational direction, the specified non-linear stiffness is generated by the spring force and the normal reaction force between the two links. Thus, this kinematic pair can be regarded as a revolute pair with a flexible translational constraint which can achieve both flexibility and rigidity with the non-linear stiffness.

In order to synthesize a practical human safe mechanism, flexibility in multiple directions is required. However, many FCRPs are required to achieve flexibility in multiple directions because the previous FCRP has flexibility just in a single direction. Thus, the whole mechanism cannot be simplified. In order to solve this problem, the FCRP with flexibility in multiple directions is proposed. This type of the FCRP is called the multi-directionally FCRP (MFCRP). Fig.2 shows the 
structure to achieve the MFCRP. It has a link with two spherical surfaces and a link with two cam surfaces. Each set of the spherical surface and the cam surface is kept in contact at a single point by using two linear springs arranged between the two links. If the two links keep in contact at the two points with each other, this kinematic pair has 4 DOF. Note that an endpoint of each spring is attached on the axis passing through two center points of the spheres, and multi-axial free rotations are allowed at the attached point. Thus, this kinematic pair has zero stiffness just around the axis passing through center points of the two spheres. This axis is called the main-rotation axis bellow. In other words, the two links are easy to rotate relatively around the main-rotation axis and hard to move in the other directions of relative motions (but it is not rigid). Therefore, this kinematic pair can be regarded as a revolute pair which has flexible constraints in the multiple directions of motions. Besides, this kinematic pair can have the specified non-linear stiffness generated by the spring forces and reaction forces between the two links in the two relative translational directions. In this paper, the design methodology of the MFCRP to implement the specified non-linear stiffness in the relative translational directions between the two links is proposed and examined. Besides, a simple flexible mechanism with flexibility in the multiple directions is synthesized with the MFCRP, and its flexibility and kinematic performances are investigated through some experiments.

In section 2, the design methodology of the MFCRP is described. In section 3, some design examples of the MFCRP are shown. In section 4, the examples are fabricated and tested to confirm the validation of the proposed design methodology. In section 5, a simple link mechanism with the MFCRP and its performances are investigated through some experiments.

\section{Design methodology}

In this section, the design methodology of the cam surface of the MFCRP to implement the specified non-linear stiffness in the relative translational directions between its two links is described. Fig.3 shows a set of the spherical surface and the cam surface kept in contact at a point. $\Sigma_{1}$ is the reference frame fixed in the link with the cam surface (link1). $\Sigma_{2}$ is the reference frame fixed in the link with the spherical surface (link2). Two same linear springs are arranged between the two links, whose endpoints $\mathrm{B}_{1}$ and $\mathrm{B}_{2}$ are attached on the main-rotation axis on the link2 with free rotations around two axes. The other endpoints $\mathrm{A}_{1}$ and $\mathrm{A}_{2}$ are attached to the link1 with free rotation around two axes. Note that considering one pair of the spherical surface and the cam surface is sufficient in the design procedure although the MFCRP has the two pairs of them because the function of the two pairs is equivalent.

As the non-linear stiffness implemented in $x_{1}$ and $y_{1}$ directions, the following two types of force-displacement characteristics can be considered as in the design of the previous FCRP (Kimura et al., 2019).

(a) Relationship between external force and displacement of the center of the sphere

(b) Relationship between external force and displacement of the contact point between the two surfaces

When the characteristics (a) are used, designers can understand the motion when an external force is applied to the MFCRP intuitively. However, the size of the cam surface may be large because it cannot be controlled in the design process. On the other hand, when the characteristics (b) are used, designers can control the size of the cam surface because the cam

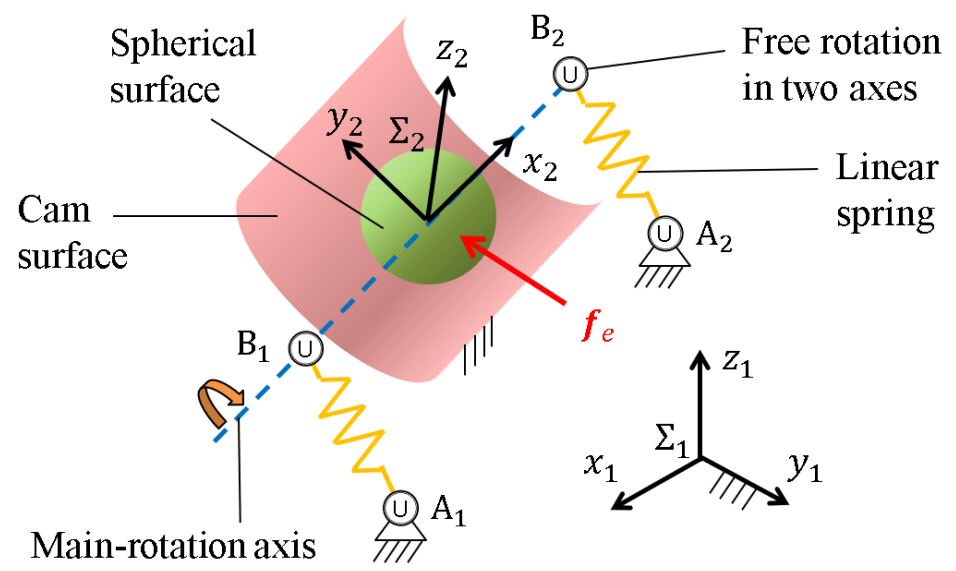

Fig. 3 Geometrical model of the MFCRP for the design methodology 
surface is the same as the area where the contact point can move. However, the relative displacement of the two links is not intuitive for designers. Since the advantage and the disadvantage of the characteristics (a) and (b) are opposite, one of the two characteristics should be chosen by designers according to its application. If the intuitive design is required, the characteristics (a) are chosen. If a compact size joint is required, the characteristics (b) are chosen. This section describes the design methodology of the cam surface to implement each of the force-displacement characteristics.

\subsection{Design based on the center of the sphere}

When an external force ${ }^{1} \boldsymbol{f}_{e}=\left[f_{e, x_{1}} f_{e, y_{2}} 0\right]^{T}$ is applied to the sphere, the following equation holds from the principal of the virtual work.

$$
f_{e, x_{1}} d x_{1}+f_{e, y_{1}} d y_{1}=\sum_{i=1}^{2}\left(k s_{i}+w_{0}\right) d s_{i}
$$

where $k$ is spring constant, $s_{i}$ is displacement of the $i$-th linear spring and $w_{0}$ is initial tension of linear springs. $s_{i}$ is calculated with the following equation.

$$
s_{i}=\left\|\left({ }^{1} \boldsymbol{R}_{2}{ }^{2} \boldsymbol{b}_{i}+{ }^{1} \boldsymbol{p}_{c}\right)-{ }^{1} \boldsymbol{a}_{i}\right\|-l_{0},
$$

where ${ }^{1} \boldsymbol{R}_{2}$ is the rotation matrix from $\Sigma_{1}$ to $\Sigma_{2},{ }^{1} \boldsymbol{a}_{i}$ is the position vector of $\mathrm{A}_{\mathrm{i}}$ in $\Sigma_{1},{ }^{2} \boldsymbol{b}_{i}$ is the position vector of $\mathrm{B}_{\mathrm{i}}$ on $\Sigma_{2},{ }^{1} \boldsymbol{p}_{c}$ is the position vector of the center of the sphere on $\Sigma_{1}$ and $l_{0}$ is initial length of the linear springs. Note that the MFCRP allows two-axial relative rotations as well as 2 -axial relative translations. Thus, ${ }^{1} \boldsymbol{R}_{2}$ can be represented as ${ }^{1} \boldsymbol{R}_{2}\left(\theta_{r}, 0, \theta_{y}\right)$, where $\theta_{r}$ is the roll angle around $x_{2}$ axis and $\theta_{y}$ is the yaw angle around $z_{2}$ axis. The pitch angle $\theta_{p}$ around $y_{2}$ axis is fixed to zero. In this design procedure, a set of posture angles $\left(\theta_{r}, \theta_{y}\right)$ is specified, and it is assumed that the two links move in the relative translational directions while maintaining that posture angles. Then, both sides of Eq.(1) are integrated and the following design equation is derived.

$$
\sum_{i=1}^{2}\left[\frac{k}{2}\left(s_{i}^{2}-s_{i, 0}^{2}\right)+w_{0}\left(s_{i}-s_{i, 0}\right)\right]-\int_{u_{0}}^{u} f_{e, x_{1}} d x_{1}-\int_{v_{0}}^{v} f_{e, y_{1}} d y_{1}=0,
$$

where $s_{i, 0}$ is the initial displacement of the $i$-th linear spring. The desired force displacement characteristics ${ }^{1} \boldsymbol{f}_{e}=$ $\left[f_{e, x_{1}} f_{e, y_{1}} 0\right]^{T}=\left[f_{d, x_{1}}\left(x_{1}, y_{1}\right) f_{d, y_{1}}\left(x_{1}, y_{1}\right) 0\right]^{T}$ is substituted into Eq.(3) and is then solved for ${ }^{1} \boldsymbol{p}_{c}$. Then, the surface which the center of the sphere path through is derived. When ${ }^{1} \boldsymbol{p}_{c}$ is represented as ${ }^{1} \boldsymbol{p}_{c}=\left[\begin{array}{lll}x_{1} & y_{1} & z_{1}\end{array}\right]^{T}$, the surface can be calculated by solving Eq.(3) for $z_{1}$ about each $\left(x_{1}, y_{1}\right)$ by using a numerical method such as the Newton method. A unit normal vector of this surface $z_{1}=g_{c}\left(x_{1}, y_{1}\right)$ is calculated with the following equation.

$$
{ }^{1} \boldsymbol{n}_{c}=\frac{1}{\sqrt{1+\left(\frac{\partial g_{c}}{\partial x_{1}}\right)^{2}+\left(\frac{\partial g_{c}}{\partial y_{1}}\right)^{2}}}\left[\begin{array}{l}
\frac{\partial g_{c}}{\partial x_{1}} \\
\frac{\partial g_{c}}{\partial y_{1}} \\
-1
\end{array}\right]
$$

Therefore, the profile of the cam surface can be calculated with the following equation.

$$
{ }^{1} \boldsymbol{p}_{s}\left(x_{1}, y_{1}\right)={ }^{1} \boldsymbol{p}_{c}\left(x_{1}, y_{1}\right)+r{ }^{1} \boldsymbol{n}_{c},
$$

where $r$ is radius of the sphere. Finally, the two same cam surface ${ }^{1} \boldsymbol{p}_{s}\left(x_{1}, y_{1}\right)$ are arranged in parallel in $\Sigma_{1}$ in order to obtain the whole cam surface shown in Fig.2.

\subsection{Design based on the contact point}

Let the cam surface be $z_{a}=g_{s}\left(x_{1}, y_{1}\right)$. Then, a unit normal vector of the cam surface in $\Sigma_{1}$ is calculated with the following equation.

$$
{ }^{1} \boldsymbol{n}_{s}=\frac{1}{\sqrt{1+\left(\frac{\partial g_{s}}{\partial x_{1}}\right)^{2}+\left(\frac{\partial g_{s}}{\partial y_{1}}\right)^{2}}}\left[\begin{array}{c}
-\frac{\partial g_{s}}{\partial x_{1}} \\
-\frac{\partial g_{s}}{\partial y_{1}} \\
1
\end{array}\right]
$$

By using $\boldsymbol{n}_{s}$, the relative position between $\mathrm{A}_{\mathrm{i}}$ and $\mathrm{B}_{\mathrm{i}}$ is calculated with the following equation.

$$
{ }^{1} \boldsymbol{d}_{i}=\left({ }^{1} \boldsymbol{R}_{2}{ }^{2} \boldsymbol{b}_{i}+{ }^{1} \boldsymbol{p}_{c}+r^{1} \boldsymbol{n}_{s}\right)-{ }^{1} \boldsymbol{a}_{i}
$$


Thus, the summation of the two spring forces are calculated with the following equation.

$$
{ }^{1} \boldsymbol{w}=-\sum_{i=1}^{2}\left[k\left(\left\|{ }^{1} \boldsymbol{d}_{i}\right\|-l_{0}\right)+w_{0}\right] \frac{{ }^{1} \boldsymbol{d}_{i}}{\left\|{ }^{1} \boldsymbol{d}_{i}\right\|}
$$

When an external force ${ }^{1} \boldsymbol{f}_{e}=\left[f_{e, x_{1}} f_{e, y_{1}} 0\right]^{T}$ is applied to the sphere, the following statics equation holds.

$$
{ }^{1} \boldsymbol{f}_{e}+{ }^{1} \boldsymbol{w}+{ }^{1} \boldsymbol{f}_{n}=\mathbf{0},
$$

where ${ }^{1} f_{n}$ is the normal force applied from the cam surface to the spherical surface and it is represented as the following equation with Eq.(6).

$$
{ }^{1} \boldsymbol{f}_{n}=\alpha^{1} \boldsymbol{n}_{s}=\frac{\alpha}{\sqrt{1+\left(\frac{\partial g_{s}}{\partial x_{1}}\right)^{2}+\left(\frac{\partial g_{s}}{\partial y_{1}}\right)^{2}}}\left[\begin{array}{c}
-\frac{\partial g_{s}}{\partial x_{1}} \\
-\frac{\partial g_{s}}{\partial y_{1}} \\
1
\end{array}\right](\alpha \in \mathbb{R})
$$

Let ${ }^{1} \boldsymbol{w}$ be ${ }^{1} \boldsymbol{w}=\left[w_{x_{1}} w_{y_{1}} w_{z_{1}}\right]^{T}$. Then, the following derivative equations are derived from Eq.(9) and Eq.(10).

$$
\left\{\begin{array}{l}
w_{z_{1}} \frac{\partial g_{s}}{\partial x_{1}}+f_{e, x_{1}}+w_{x_{1}}=0 \\
w_{z_{1}} \frac{\partial g_{s}}{\partial y_{1}}+f_{e, y_{1}}+w_{y_{1}}=0
\end{array}\right.
$$

The desired force displacement characteristics ${ }^{1} \boldsymbol{f}_{e}=\left[f_{e, x_{1}} f_{e, y_{1}} 0\right]^{T}=\left[f_{d, x_{1}}\left(x_{1}, y_{1}\right) f_{d, y_{1}}\left(x_{1}, y_{1}\right) 0\right]^{T}$ is substituted into Eq.11 and it is solved for $z_{1}=g_{s}\left(x_{1}, y_{1}\right)$. Note that it is difficult to solve the derivative equation because $\frac{\partial g_{s}}{\partial x_{1}}$ and $\frac{\partial g_{s}}{\partial y_{1}}$ are implicitly included in $w_{x_{1}} w_{y_{1}} w_{z_{1}}$. Thus, this differential equation is solved with the following procedure.

(1) $\left(x_{1}, y_{1}, z_{1}\right)$ is substituted into Eq.(11).

(2) Eq.(11) is assumed as a non-linear equations for $\frac{\partial g_{s}}{\partial x_{1}}$ and $\frac{\partial g_{s}}{\partial x_{1}}$, and solved for them with a numerical method such as the Newton-Raphson method.

(3) $z_{1}$ is updated with the following equations.

$$
\begin{aligned}
& z_{1}\left(x_{1}+h, y_{1}\right)=z_{1}\left(x_{1}, y_{1}\right)+\frac{\partial g_{s}}{\partial x_{1}} h \\
& z_{1}\left(x_{1}, y_{1}+h\right)=z_{1}\left(x_{1}, y_{1}\right)+\frac{\partial g_{s}}{\partial y_{1}} h \\
& z_{1}\left(x_{1}+h, y_{1}+h\right)=z_{1}\left(x_{1}, y_{1}\right)+\frac{\partial g_{s}}{\partial x_{1}} h+\frac{\partial g_{s}}{\partial y_{1}} h
\end{aligned}
$$

where $h$ is a step variable.

The above procedure (1)-(3) is performed for each position of $\left(x_{1}, y_{1}\right)$. The whole cam surface shown in Fig.2 can be obtained by arranging two same cam surface $z_{1}=g_{s}\left(x_{1}, y_{1}\right)$ in parallel in $\Sigma_{1}$.

\section{Design examples}

In this section, several examples of cam surfaces are shown. They were designed to have specified non-linear stiffness based on the displacement of the center of the sphere and the contact point between the two surfaces.

\subsection{Example based on the center of the sphere}

Design parameters are listed in Table1, where $x_{1}$ axis is in parallel to the main-rotational axis of the MFCRP. The specified force-displacement characteristics shown in Table1 are illustrated in Fig.4. The figure (a) shows the specified characteristics in the $x_{1}$-direction and (b) shows the characteristics in the $y_{1}$-direction. These characteristics are hardening spring characteristics.

The cam surface designed with the method in section 2.1 is shown in Fig.5. In order to simplify the design problem, just a part of a cam surface in $x_{1} \geqq 0$ and $y_{1} \geqq 0$ was designed as shown in Fig.5 (a). The width of the calculated cam surface was about $15 \mathrm{~mm}$ in the $x_{1}$-direction and about $20 \mathrm{~mm}$ in the $y_{1}$-direction. These sizes were bigger than the specified displacement $10 \mathrm{~mm}$. One of two cam surfaces shown in Fig. 2 was obtained by placing this surface symmetrically about $x_{1}-z_{1}$ plane, $y_{1}-z_{1}$ plane and $z_{1}$ axis. Then, two of the cam surface were placed next to each other so that the origin of $\Sigma_{1}$ was located at $(x, y, z)=(16,0,0),(-16,0,0)$ on the $\mathrm{O}-x y z$ coordinates as shown in Fig.5 (b). 
Kimura, Iwatsuki and Ikeda, Mechanical Engineering Journal, Vol.7, No.4 (2020)

Table 1 Design parameters for the examples with relationship between external force and displacement of the center point of the sphere

\begin{tabular}{|l|c|}
\hline Stiffness in the $x_{1}$-direction $f_{d, x_{1}}\left(x_{1}, y_{1}\right)[\mathrm{N}]$ & $3.0\left(e^{0.1 x_{1}}-1\right)\left(0 \leqq x_{1} \leqq 10\right)$ \\
\hline Stiffness in the $y_{1}$-direction $f_{d, y_{1}}\left(x_{1}, y_{1}\right)[\mathrm{N}]$ & $6.0\left(e^{0.1 y_{1}}-1\right)\left(0 \leqq y_{1} \leqq 10\right)$ \\
\hline Spring constant $k[\mathrm{~N} / \mathrm{mm}]$ & 0.166 \\
\hline Natural length of springs $l_{0}[\mathrm{~mm}]$ & 20 \\
\hline Initial tension of springs $w_{0}[\mathrm{~N}]$ & 1 \\
\hline Relative posture (roll pitch yaw angle $)[\mathrm{rad}]$ & $\left(\theta_{r}, \theta_{p}, \theta_{y}\right)=(0,0,0)$ \\
\hline End points of springs on pairing element $1[\mathrm{~mm}]$ & $\mathrm{A}_{1}:(30,0,0), \mathrm{A}_{2}:(-60,0,0)\left(\right.$ on $\left.\Sigma_{1}\right)$ \\
\hline End points of springs on pairing element $2[\mathrm{~mm}]$ & $\mathrm{B}_{1}:(30,0,0), \mathrm{B}_{2}:(-60,0,0)\left(\right.$ on $\left.\Sigma_{2}\right)$ \\
\hline Radius of the sphere $r[\mathrm{~mm}]$ & 13 \\
\hline Initial position $\left(x_{1,0}, y_{1,0}, z_{1,0}\right)[\mathrm{mm}]$ & $(0,0,33)$ \\
\hline
\end{tabular}

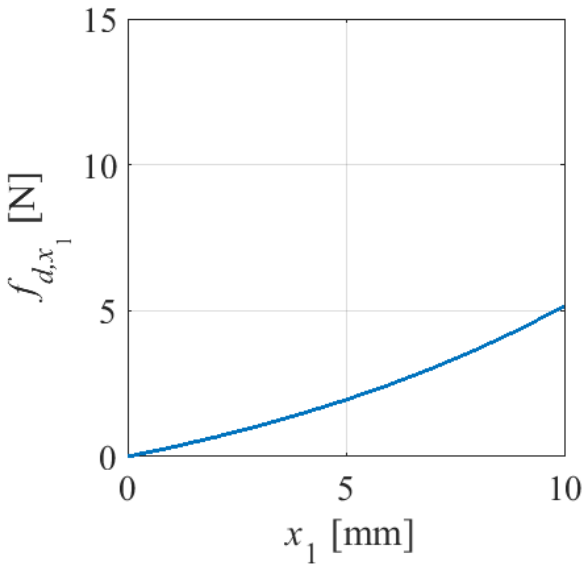

(a) $x_{1}$-direction

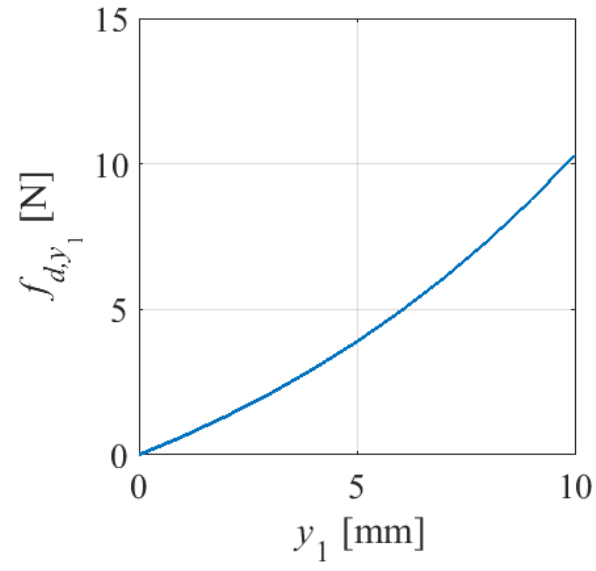

(b) $y_{1}$-direction

Fig. 4 The specified relationships between external force and displacement of the center point of the sphere

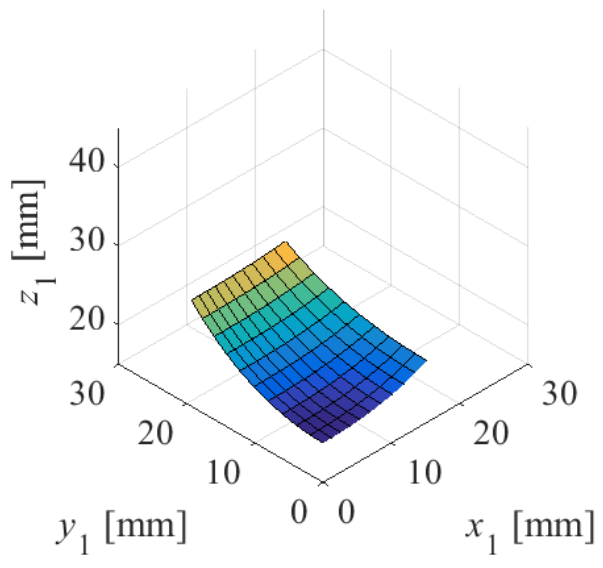

(a) Calculated surface

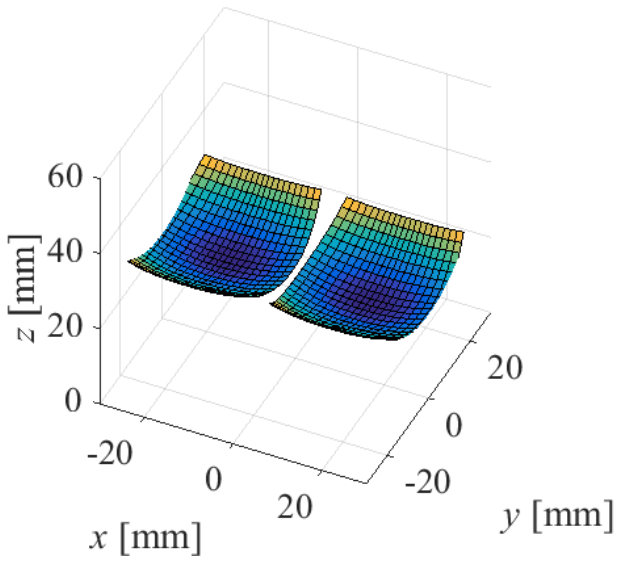

(b) Whole cam surface

Fig. 5 The calculated cam surfaces designed with relationships between external force and displacement of the center point of the sphere

\subsection{Example based on the contact point}

Design parameters are listed in Table2, where the $x_{1}$-direction is in parallel to the main-rotation axis of the MFCRP. The specified force-displacement characteristics are shown in Fig.6. These characteristics are hardening spring character- 
Kimura, Iwatsuki and Ikeda, Mechanical Engineering Journal, Vol.7, No.4 (2020)

Table 2 Design parameters for the example with relationships between external force and displacement of the contact point

\begin{tabular}{|l|c|}
\hline Stiffness in the $x_{1}$-direction $f_{d, x_{1}}\left(x_{1}, y_{1}\right)[\mathrm{N}]$ & $1.5\left(e^{0.1 x_{1}}-1\right)\left(0 \leqq x_{1} \leqq 15\right)$ \\
\hline Stiffness in the $y_{1}$-direction $f_{d, y_{1}}\left(x_{1}, y_{1}\right)[\mathrm{N}]$ & $2.0\left(e^{0.1 y_{1}}-1\right)\left(0 \leqq y_{1} \leqq 15\right)$ \\
\hline Spring constant $k[\mathrm{~N} / \mathrm{mm}]$ & 0.166 \\
\hline Natural length of springs $l_{0}[\mathrm{~mm}]$ & 20 \\
\hline Initial tension of springs $w_{0}[\mathrm{~N}]$ & 1 \\
\hline Relative posture (roll pitch yaw angle) $[\mathrm{rad}]$ & $\left(\theta_{r}, \theta_{p}, \theta_{y}\right)=(0,0,0)$ \\
\hline End points of springs on pairing element $1[\mathrm{~mm}]$ & $\mathrm{A}_{1}:(25,0,0), \mathrm{A}_{2}:(-55,0,0)\left(\right.$ on $\left.\Sigma_{1}\right)$ \\
\hline End points of springs on pairing element $2[\mathrm{~mm}]$ & $\mathrm{B}_{1}:(25,0,0), \mathrm{B}_{2}:(-55,0,0)\left(\right.$ on $\left.\Sigma_{2}\right)$ \\
\hline Radius of the sphere $r[\mathrm{~mm}]$ & 13 \\
\hline Initial position $\left(x_{1,0}, y_{1,0}, z_{1,0}\right)[\mathrm{mm}]$ & $(0,0,20)$ \\
\hline
\end{tabular}

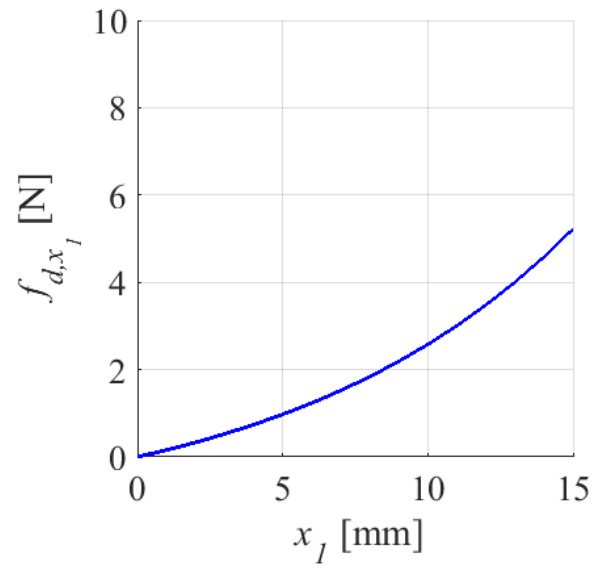

(a) $x_{1}$-direction

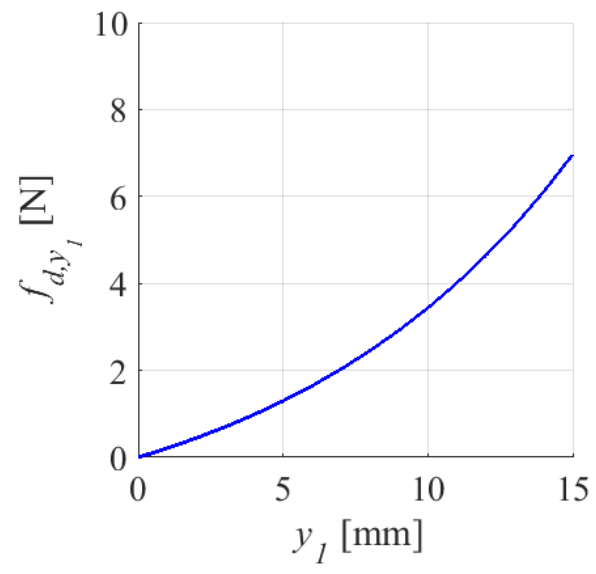

(b) $y_{1}$-direction

Fig. 6 The specified relationships between external force and displacement of the contact point between two links

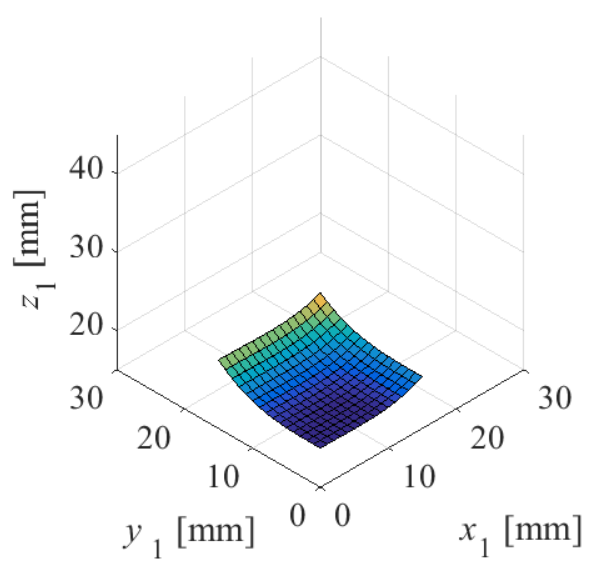

(a) Calculated surface

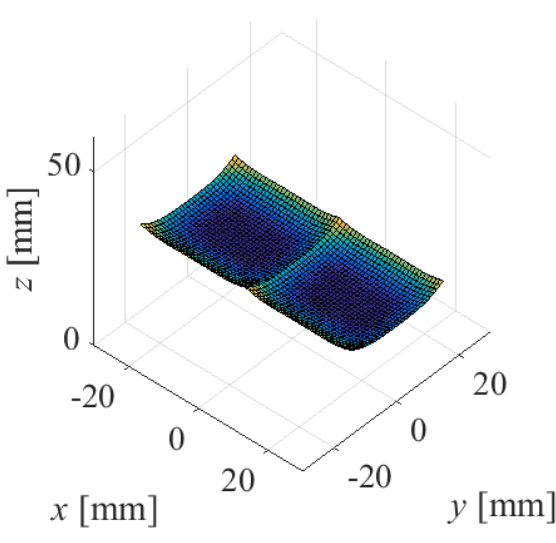

(b) Whole cam surface

Fig. 7 The calculated cam surfaces designed with relationships between external force and displacement of the contact point between two links

istics. The coordinates $x_{1}$ and $y_{1}$ in these characteristics represent the displacement of the contact point between the cam surface and the spherical surface from the origin of $\Sigma_{1}$.

Fig.7 (a) shows the calculated a part of the cam surface in $x_{1} \geqq 0$ and $y_{1} \geqq 0$. Then, a cam surface was obtained by 
placing this part symmetrically about $x_{1}-z_{1}$ plane, $y_{1}-z_{1}$ plane and $z_{1}$ axis. Finally, two of the cam surface were placed so that the origin of $\Sigma_{1}$ was located at $(x, y, z)=(15,0,0),(-15,0,0)$ on the $\mathrm{O}-x y z$ coordinates as shown in Fig.7 (b). The width of the calculated part of the surface shown in Fig7 (a) was $15 \mathrm{~mm}$ in both $x_{1}$ - and $y_{1}$-direction. This was the same value of the specified displacement shown in Table2. Therefore, the size of the cam surface was able to be controlled with this method although the relative displacement between the two links is not intuitive for designers.

\section{Prototyping and evaluation}

In this section, some prototypes of the MFCRP are fabricated and experimentally examined. The prototype with the cam surface designed in section 3.1 was fabricated by a 3D-printer with the fused deposition modeling (FDM) as shown in Fig.8. Its motion range of the main-rotation was about $-\frac{\pi}{2} \leqq \theta_{r} \leqq \frac{\pi}{2}$ [rad]. Besides, the prototype with the cam surface designed in section 3.2 was fabricated by a 3D-printer with the material jetting. This prototype has the same structure as the prototype shown in Fig.8. However, the modeling accuracy of the prototype fabricated by the material jetting is better than the prototype fabricated by the FDM. By comparing the MFCRPs fabricated by the two different methods, it is possible to investigate the effect of modeling accuracy on the experimental results.

The force-displacement characteristics of the prototypes in the $x$ - and $y$-directions shown in Fig.8 were measured with a force gauge. Fig.9 shows the experimental setup. The force-displacement characteristics in the $x$ - and $y$-directions are measured by the tensile testing machine composed of the force gauge and a stand for the force gauge. The prototype

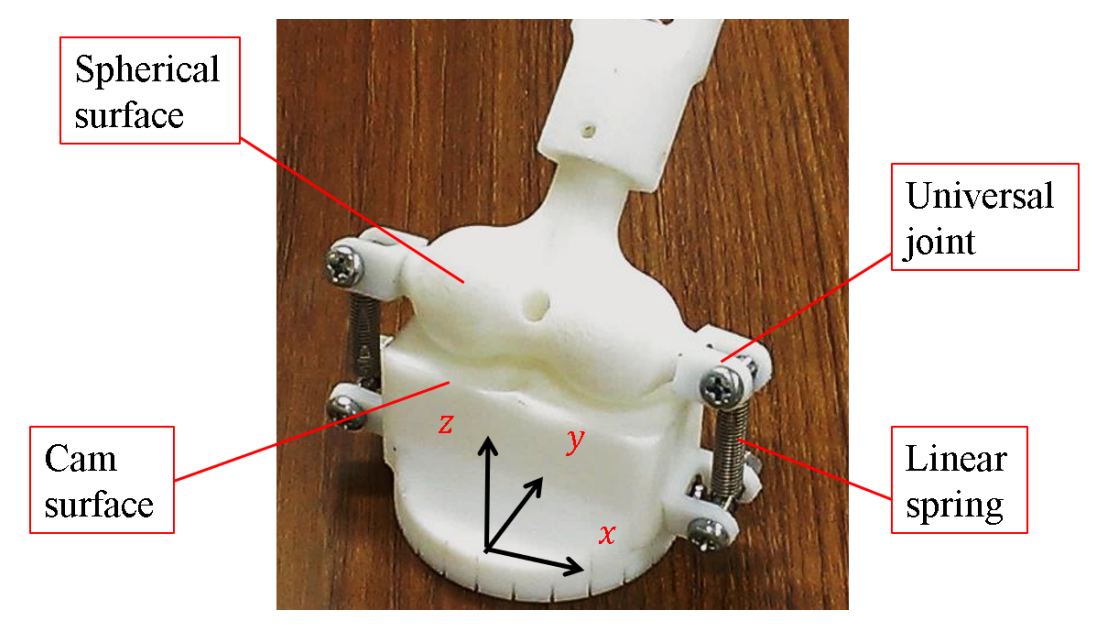

Fig. 8 Prototype of the MFCRP fabricated by a 3D-printer

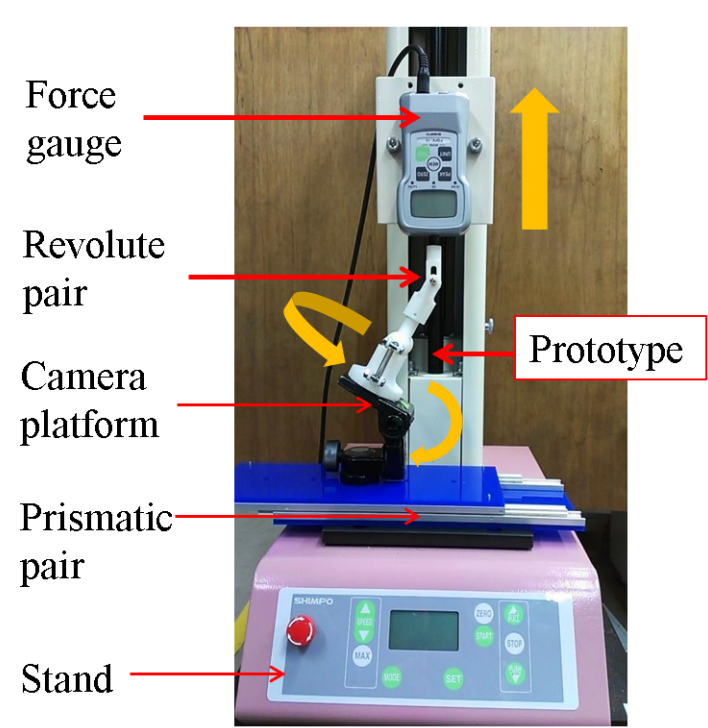

(a) Overall view

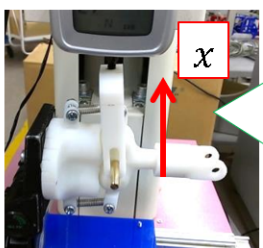

(b) $x$-direction

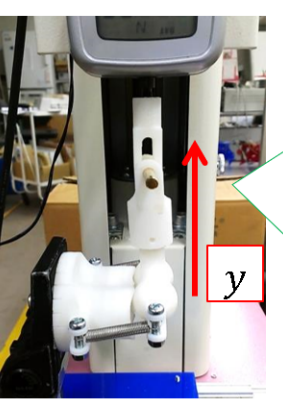

(c) $y$-direction

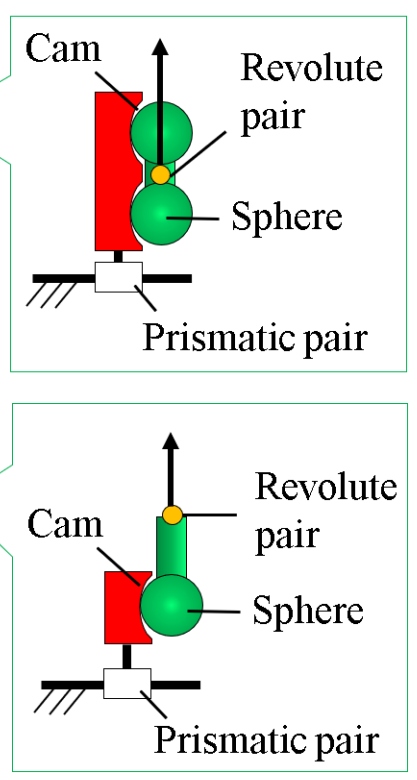

Fig. 9 Experimental setup to measure force-displacement characteristics between two links of prototypes 
mounted on a camera platform was attached to the force-gauge stand through prismatic pair to reduce loads orthogonal to the measured direction. In the measurement, the link is connected to the force gauge via a revolute pair as shown in Fig.9 (b) and (c) so that the force acts at midpoint between the two spheres. In order to reduce friction as much as possible, a lubricant is applied between the two links of the MFCRP.

Fig.10 shows force-displacement characteristics of the prototype with the cam surface designed in section 3.1. Fig.10 (a) shows the result of the $x$-direction and Fig.10 (b) shows the result of the $y$-direction. The blue solid line in each figure is the measured characteristics and the green dotted line is the calculated theoretical characteristics. These figures show that the measured characteristics were little different from the calculated characteristics. It is considered that these errors were caused by friction between the two surfaces because the friction between the two links was not able to be small enough. Thus, the theoretical characteristics were modified to include the effect of the friction with the following equation.

$$
{ }^{1} \boldsymbol{f}_{d}=-{ }^{1} \boldsymbol{w}+\frac{{ }^{1} \boldsymbol{e}_{z, 1}^{T}{ }^{1} \boldsymbol{w}}{{ }^{1} \boldsymbol{e}_{z, 1}^{T}\left({ }^{1} \boldsymbol{n}_{s}-\mu^{1} \boldsymbol{v}\right)}\left({ }^{1} \boldsymbol{n}_{s}-\mu^{1} \boldsymbol{v}\right),
$$

where $\mu$ is the coefficient of static friction, ${ }^{1} \boldsymbol{v}$ is the unit tangent vector of the cam surface in the direction of the motion

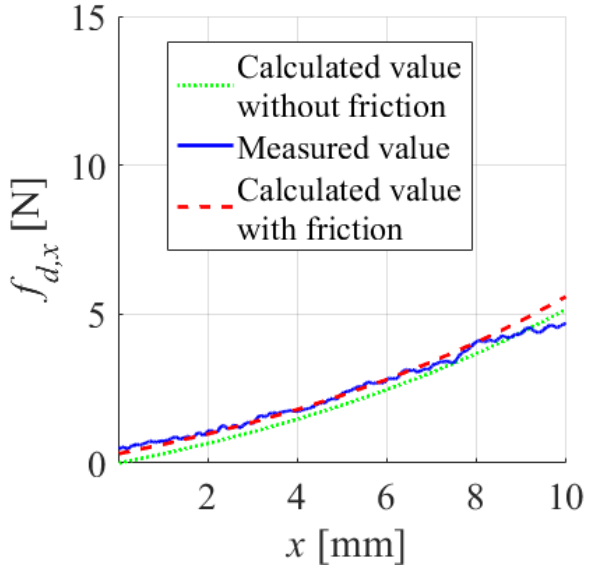

(a) $x_{1}$-direction

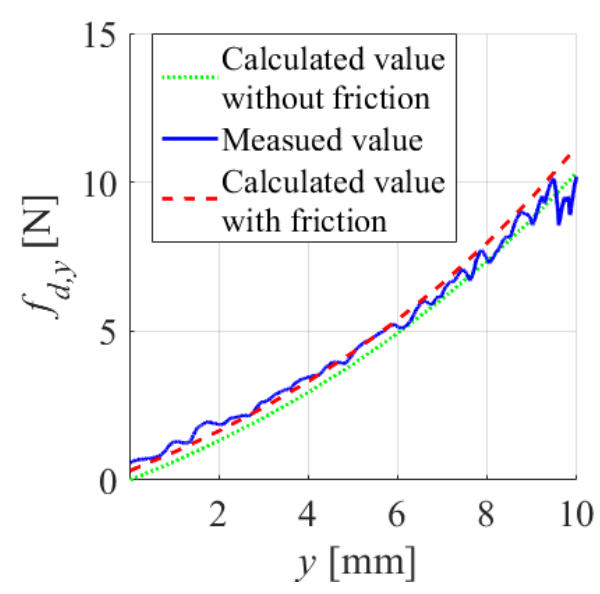

(b) $y_{1}$-direction

Fig. 10 Comparison between calculated and measured stiffness characteristics of the prototype with the cam surface designed in section 3.1

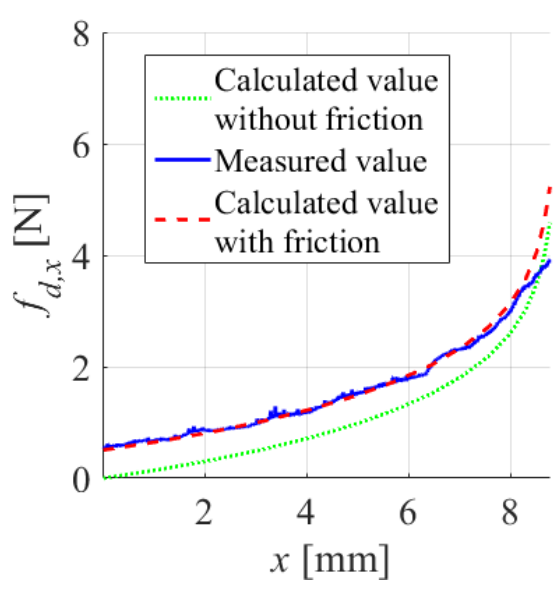

(a) $x_{1}$-direction

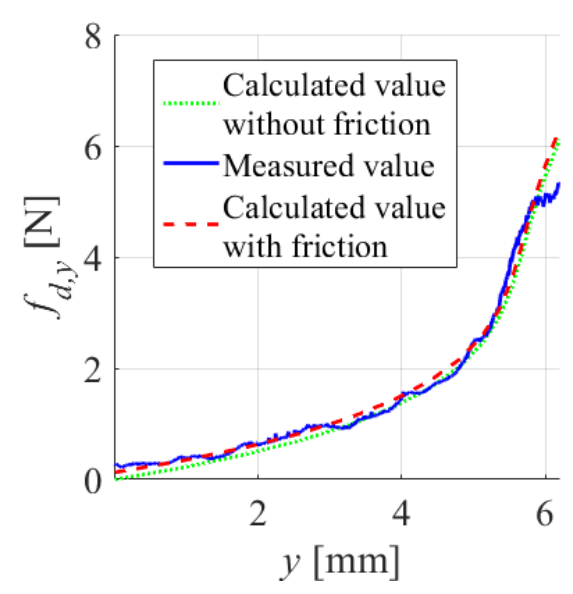

(b) $y_{1}$-direction

Fig. 11 Comparison between calculated and measured stiffness characteristics of the prototype with the cam surface designed in section 3.2 
and ${ }^{1} \boldsymbol{e}_{z, 1}$ is the unit vector in the $z_{1}$-direction. The coefficient of static friction was substituted into Eq.(15) so that the theoretical characteristics fit the measured ones because measuring the coefficient of the static friction on the curved surface is difficult. The recalculated theoretical values are shown as the red broken lines in Fig.10 (a) and (b). The calculated characteristics agreed very well with the measured ones. Note that the coefficient of static friction in both results in the $x$ - and $y$-directions was 0.05 . This value can be agreed upon intuitively.

Fig.11 shows force-displacement characteristics of the prototype with the cam surface designed in section 3.2. Fig.11 (a) shows the result of the $x$-direction and (b) shows the result of the $y$-direction. The blue solid line in each figure is the measured characteristics, the green dotted line is the calculated theoretical characteristics without the effect of friction. Note that the theoretical characteristics are the characteristics based on the center of the sphere which is modified from the characteristics based on the contact point shown in Fig.6 in order to be compared with the measured characteristics. The red broken line is recalculated theoretical characteristics with the effect of the friction. The calculated characteristics with the effect of the friction agreed very well with the measured characteristics while the theoretical characteristics without the effect of the friction did not agree with the measured characteristics. In this case, the coefficient of the static friction in the $x$-direction was 0.08 and the coefficient in the $y$-direction was 0.02 . These values can be agreed upon intuitively.

In Fig.10 and Fig.11, there were large errors between the measured and the calculated values in near the maximum displacement. The reason is considered that contact points between the two links were located at the edge of the cam surface. Since the edge had a corner, the link with spherical surfaces was able to have several postures. Thus, the cause of the error is considered that the link with the spherical surface had a different posture from the theoretical one. In addition, the ripples shown in Fig.11 look less than the ripples shown in Fig.10. The reason is considered to be due to the modeling accuracy of the fabrication methods.

\section{Application}

The MFCRP allows 4 DOF, and it has zero stiffness in the main rotational direction and non-zero stiffness in the other three sub-directions. Therefore, this kinematic pair behaves like a revolute pair with 1 DOF under small external loads, while the two links of the MFCRP can displace in the other relative directions under large external loads. Therefore, a flexible underactuated linkage can easily be synthesized if ordinary passive revolute pairs in fully-actuated linkage are replaced to the MFCRPs. In this section, the flexibility and kinematic performance of a flexible linkage with the MFCRP are investigated through some experiments.

A flexible linkage with the MFCRP is shown in Fig.12. Fig.12 (a) shows the photograph of the fabricated linkage and (b) shows the size of the linkage. This linkage is a planar closed-loop five-bar linkage composed of two active revolute pairs (DC motors), two MFCRPs and one ordinary passive revolute pair. The output point is located at the passive revolute pair. The MFCRPs were designed with parameters shown in Table3 and fabricated by a 3D-printer with the FDM. Note

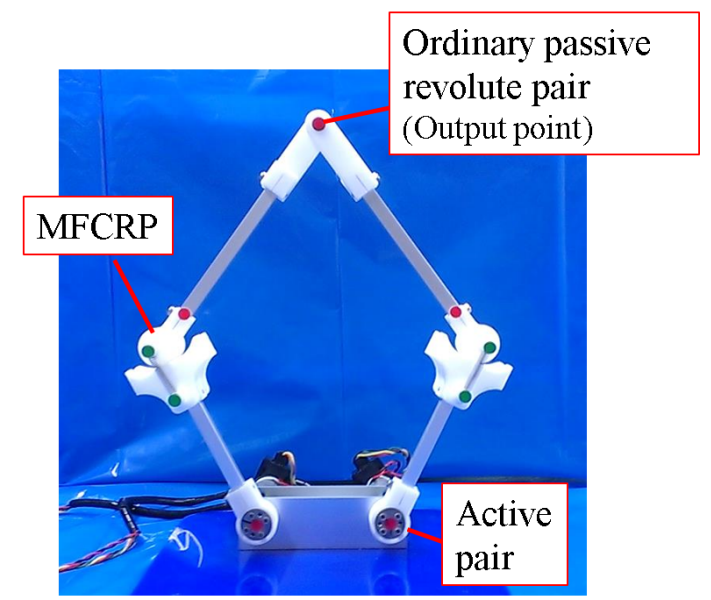

(a) Photograph of the linkage

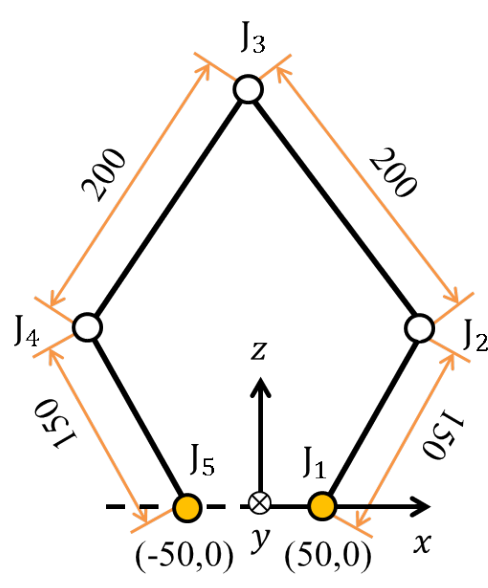

(b) Size of the linkage

Fig. 12 The fabricated planar closed-loop five-bar linkage with the MFCRPs 
Table 3 Design parameters of the MFCRP in the fabricated linkage

\begin{tabular}{|l|c|}
\hline Stiffness in the $x_{1}$-direction $f_{d, x_{1}}\left(x_{1}, y_{1}\right)[\mathrm{N}]$ & $e^{0.1 x_{1}}-1\left(0 \leqq x_{1} \leqq 15\right)$ \\
\hline Stiffness in the $y_{1}$-direction $f_{d, y_{1}}\left(x_{1}, y_{1}\right)[\mathrm{N}]$ & $e^{0.1 y_{1}}-1\left(0 \leqq y_{1} \leqq 25\right)$ \\
\hline Spring constant $k[\mathrm{~N} / \mathrm{mm}]$ & 0.110 \\
\hline Natural length of springs $l_{0}[\mathrm{~mm}]$ & 19.4 \\
\hline Initial tension of springs $w_{0}[\mathrm{~N}]$ & 0.686 \\
\hline Relative posture (roll pitch yaw angle) $[\mathrm{rad}]$ & $\left(\theta_{r}, \theta_{p}, \theta_{y}\right)=(0,0,0)$ \\
\hline End points of springs on pairing element $1[\mathrm{~mm}]$ & $\mathrm{A}_{1}:(30,0,0), \mathrm{A}_{2}:(-60,0,0)\left(\right.$ on $\left.\Sigma_{1}\right)$ \\
\hline End points of springs on pairing element $2[\mathrm{~mm}]$ & $\mathrm{B}_{1}:(30,0,0), \mathrm{B}_{2}:(-60,0,0)\left(\right.$ on $\left.\Sigma_{2}\right)$ \\
\hline Radius of the sphere $r[\mathrm{~mm}]$ & 14 \\
\hline Initial position $\left(x_{1,0}, y_{1,0}, z_{1,0}\right)[\mathrm{mm}]$ & $(0,0,25)$ \\
\hline
\end{tabular}

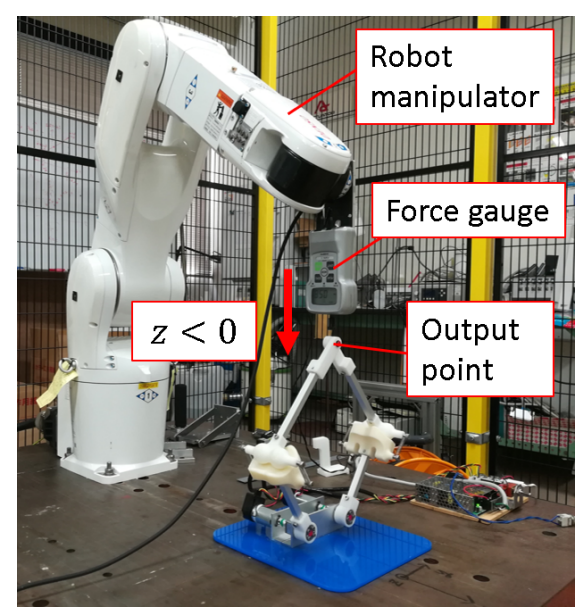

(a) z-direction

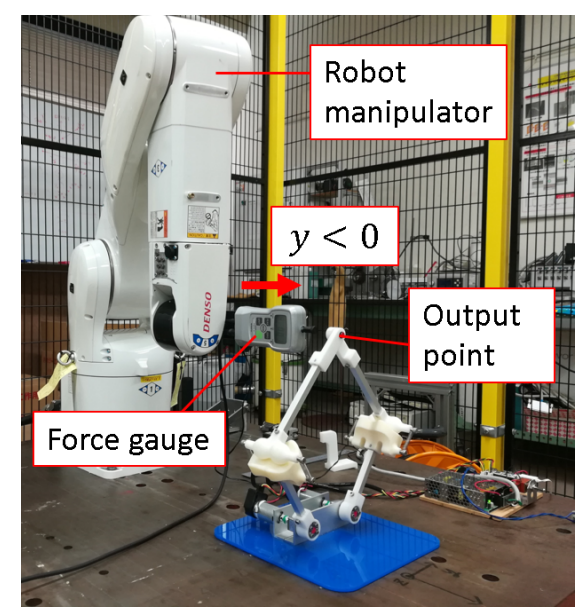

(b) $y$-direction

Fig. 13 Measurement of the force-displacement characteristics at the output point of the fabricated linkage in the multiple directions

that the motion range of the main-rotation was about $-\frac{\pi}{2} \leqq \theta_{r} \leqq \frac{\pi}{2}$ [rad]. The specified force-displacement characteristics were based on the contact point between the two surfaces of the MFCRP so that the size of the MFCRP becomes compact. In the authors' previous work, a planar closed-loop five-bar linkage with the previous FCRPs was fabricated (Kimura et al, 2019). Although the previous mechanism has flexibility only in the motion plane, the flexible linkage in this paper has a flexibility not only in the motion plane but also in the out of the motion plane because the MFCRP has flexibility in multiple directions.

\subsection{Flexibility of the output point}

In order to investigate flexibility at the output point of the linkage, the force-displacement characteristics in the $y$ - and $z$-directions shown in Fig.12 (b) were measured. Fig.13 shows the experimental setup. Force-displacement characteristics of the output point were measured by pushing the output point in the $y<0$ and $z<0$ directions with a force gauge attached to the end effector of a serial robot manipulator with 6 DOF. The rotation angle of the DC motors were controlled with the position PID control so that the output point under no load was located at $(x, y, z)=(0,0,280)[\mathrm{mm}]$.

Fig.14 shows the result of the measurement, where the red solid line in the figure (a) is the measured force-displacement characteristics in the $z$-direction and the red solid line in the figure (b) is the measured characteristics in the $y$-direction. The broken blue line in Fig.14 (a) is the theoretical characteristics which were calculated with the method described in the authors' previous work (Kimura et al., 2019). Note that the coefficient of static friction between the two links of the MFCRP was 0.05. As in the previous report, the stiffness characteristics of the DC motors were assumed as linear characteristics with the rotational servo stiffness of $1.8 \times 10^{4} \mathrm{Nmm} / \mathrm{rad}$. The output point had the hardening stiffness characteristics in the $z$-direction and the measured characteristics agreed very well with the calculated characteristics. In addition, since the linkage had the flexibility not only in the $z$-direction but also in the $y$ - direction, the flexibility in the out of the motion plane was able to be achieved with the MFCRPs. Note that the cause of the ripples in the measured 


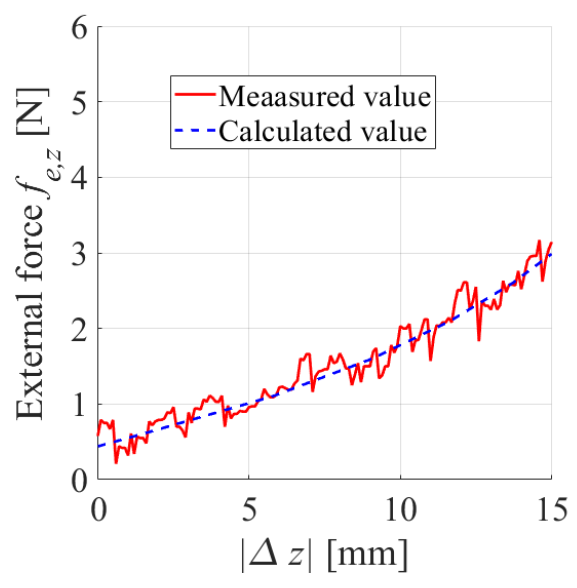

(a) $z$-direction

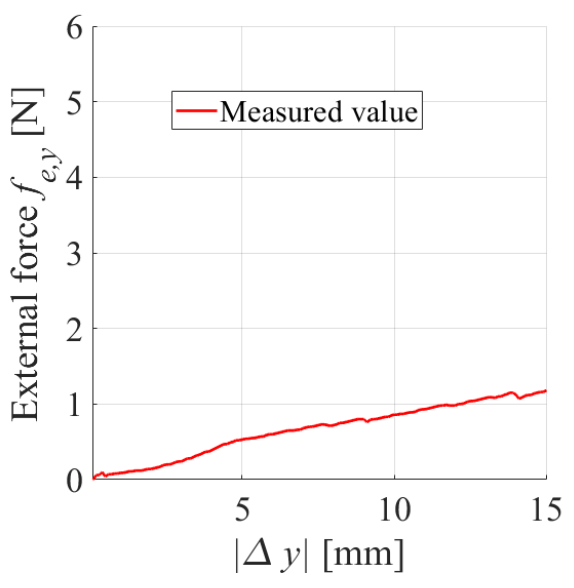

(b) $y$-direction

Fig. 14 Measured force-displacement characteristics at the output point of the fabricated flexible linkage

characteristics is considered to be due to the modeling accuracy of the FDM.

\subsection{Kinematic performance}

In order to investigate kinematic performances of the flexible linkage, the output trajectories of the linkage were measured by a motion capture system. The target trajectory was a rectangular trajectory whose vertexes are at $(x, y, z)=$ $(50,0,280),(50,0,330),(-50,0,330),(-50,0,280)[\mathrm{mm}]$. The output point generated the clockwise $(\mathrm{CW})$ and the counterclockwise $(\mathrm{CCW})$ trajectories starting from $(x, y, z)=(50,0,280)$. Input angles were calculated by the inverse kinematics of the planar five-bar closed-loop linkage where all kinematic pairs were assumed as ordinary revolute pairs. The output trajectories were measured with a stereo camera (OptiTrack V120:Duo).

Fig.15 shows the measured and target trajectories. The figure (a) shows the trajectories shown in the $x-z$ plane and (b) shows the trajectories shown in the $y-z$ plane. The green dotted line is the target trajectory, the blue solid line is the measured CCW trajectory and the red solid line is the measured CW trajectory. The measured trajectories agreed well with the specified one although the accuracy was low. Thus, it is considered that the MFCRPs in the linkage behaved like ordinary revolute pairs. Note that the measured CW trajectory was a little different from the CCW trajectory. If the error between the measured and specified trajectory was caused by the structural error of the mechanism, the two trajectories should be the same trajectories. Thus, it is considered that the error was caused by not the structural error but the displacement between the two links in each of the MFCRPs.

In Fig.15, the measured rectangular trajectories look being below the specified one. It is considered that this was because the two links in each of the MFCRPs displaced relatively due to the effect of gravity. In addition, Fig.15 (a) shows that the output point displaced in the opposite direction of the motion when the top or bottom parts of the rectangular trajectory was generated. The reason is thought to be that rolling motion between the two links in each of the MFCRPs occurred because of the friction at the contact points. Fig.16 shows the relationship between the position of the output point and time sequence of the relative angle between the two links in each of the MFCRPs when the output point generates the CCW trajectory, where the angle $\theta_{2}$ and $\theta_{4}$ are relative angle between two links of the MFCRPs, $\mathbf{J}_{2}$ and $\mathbf{J}_{4}$, respectively. When the output point generates the top part of the rectangular (in the area II in Fig.16), the rotational direction of $\theta_{2}$ is same as $\theta_{4}$. Then, the link $\mathbf{J}_{2}-\mathbf{J}_{3}$ and the link $\mathbf{J}_{3}-\mathbf{J}_{4}$ can displace in the opposite direction of the motion due to the rolling motion between the two links in each of the MFCRPs. This idea also can be applied to the case in the bottom part of the rectangular (in the area IV in Fig.16).

Next, output trajectories when an impact force applied to the output point of the linkage were measured. The specified trajectory was the CCW trajectory with the same rectangular shape starting from $(x, y, z)=(50,0,280)[\mathrm{mm}]$. This experiment was performed in the following step.

(1) The one cycle of the rectangular trajectory was measured under no external load.

(2) The outpoint was tapped by a human hand in the $z<0$ or $y<0$ directions. 


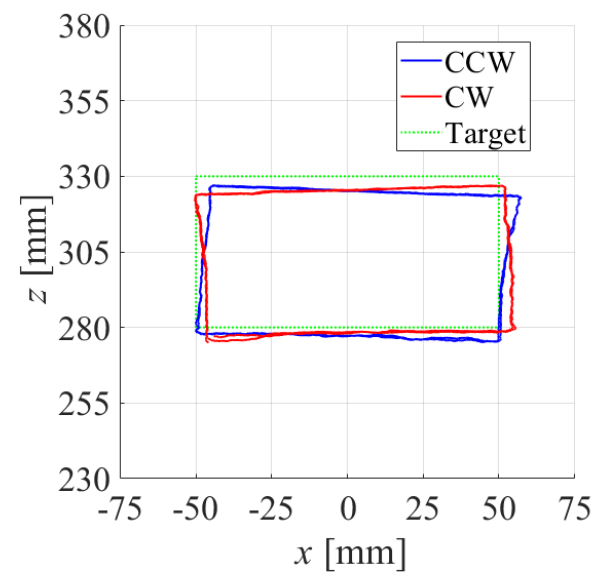

(a) $x-z$ plane

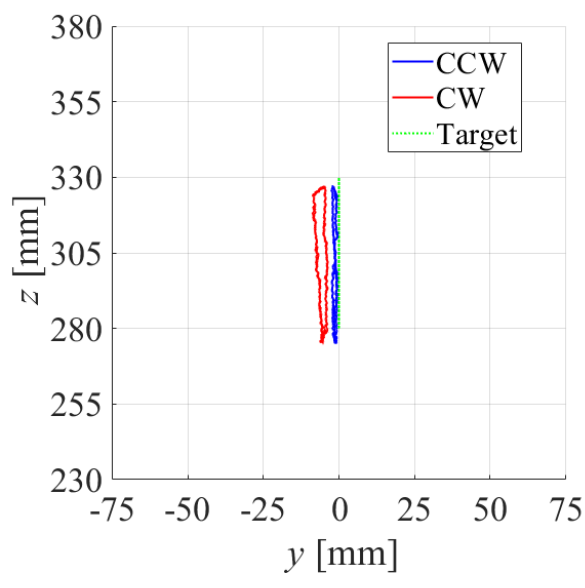

(b) $y-z$ plane

Fig. 15 The measured and specified trajectory without external load at the output point

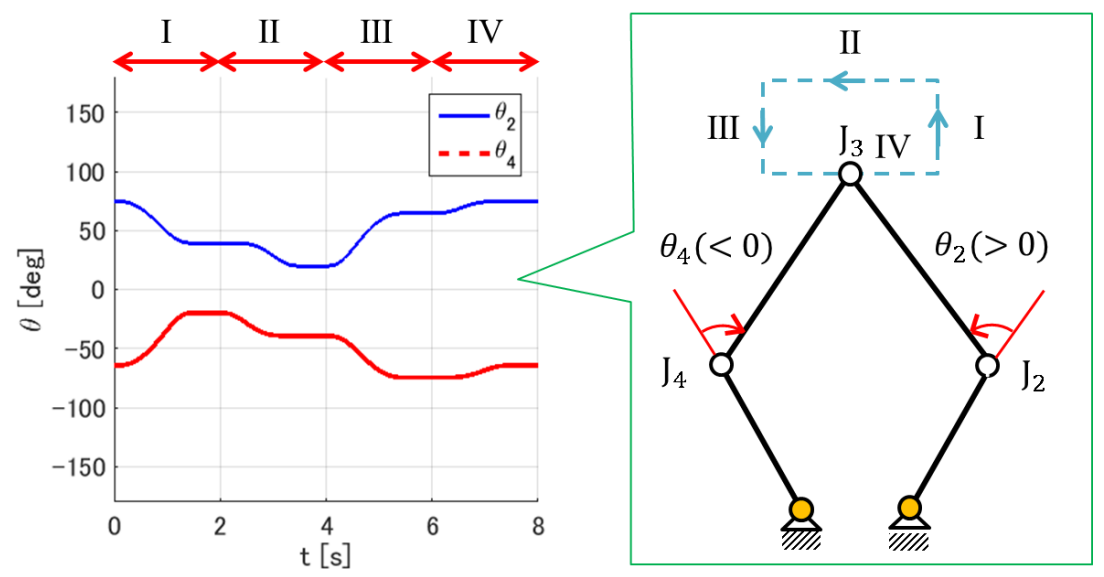

Fig. 16 Relationship between time shift of relative rotation angles of the FCRPs and the output trajectory of the fabricated linkage

(3) The one cycle of the rectangular trajectory after the tapping was measured under no external load.

Fig. 17 shows the result when the output point was tapped in the $z$-direction. The figure (a) shows the trajectories shown in the $x-z$ plane and (b) shows the trajectories shown in the $y-z$ plane. The point A shown in Fig. 17 is the position where the output point was tapped. These figures show that there were small errors between the trajectory before and after the tapping. Fig.18 shows the result when the output point was tapped in the $y$-direction. The figure (a) shows the trajectories shown in the $x-z$ plane and (b) shows the trajectories shown in the $y-z$ plane. The point A shown in Fig. 18 is the position where the output point was tapped. The trajectory after the tapping was slightly displaced to the $y$-direction. The reason is considered that the contact point between the two links in each of the MFCRPs was not able to return to the initial position completely due to the static friction. However, the effect of the friction can be reduced with enough lubrication between the two links in each of the MFCRPs. Therefore, the motion of the linkage is robust against an external load under the efficient lubrication between two links of the MFCRP.

\section{Conclusion}

In this paper, the flexibly constrained revolute pair (FCRP) which has flexibility not only in the motion plane but also in the out of the motion plane was developed. It is called the multi-directionally FCRP (MFCRP). By using this kinematic pair, a linkage with a simple structure which has flexibility in the multiple directions can be synthesized. It can have both flexibility for human safety and rigidity for force transmission by implementing the desired stiffness characteristics into 


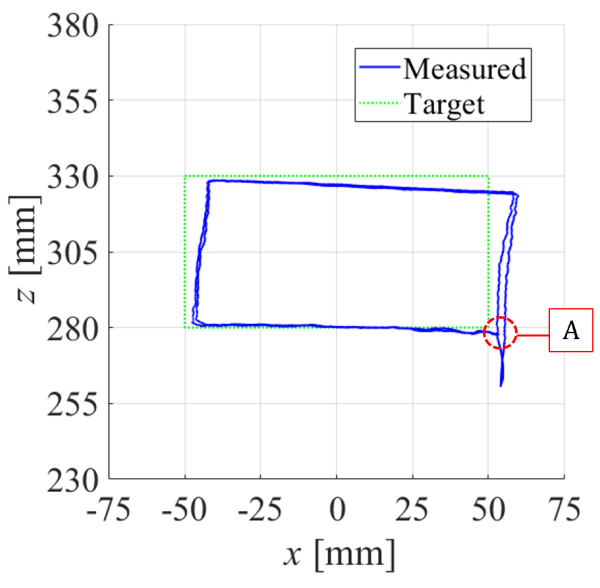

(a) $x-z$ plane

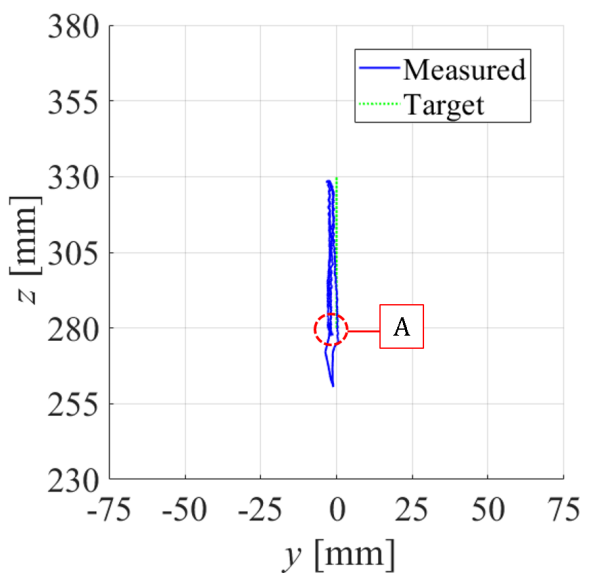

(b) $y-z$ plane

Fig. 17 The measured trajectory before and after the fabricated linkage subjected to impact force in the $z<0$ direction

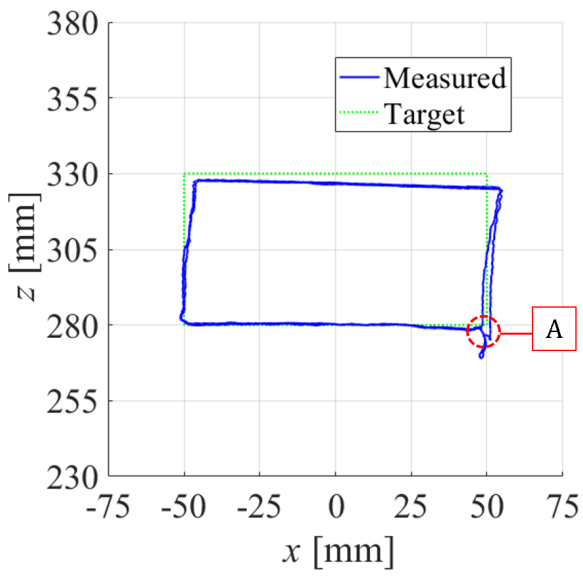

(a) $x-z$ plane

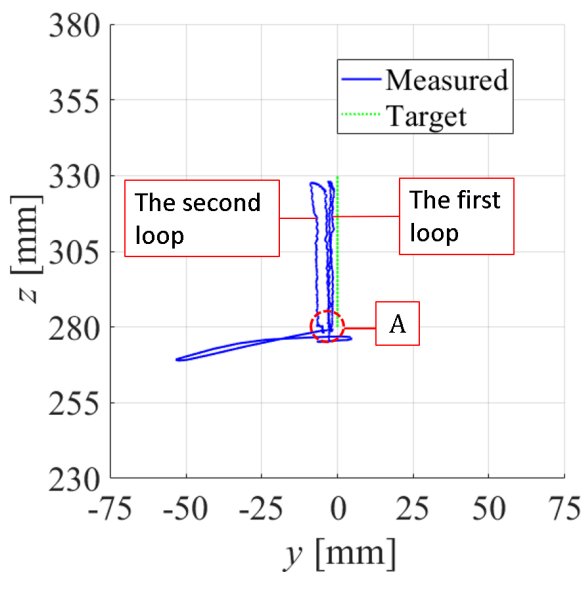

(b) $y-z$ plane

Fig. 18 The measured trajectory before and after the fabricated linkage subjected to impact force in the $y<0$ direction

the MFCRP. The results of this paper are as follows.

(1) The profile of the cam surface that generates the specified relationship between an external force and displacement of the center of the sphere is calculated by solving the energy equation between the two links. These force-displacement characteristics are intuitive for designers, although it is difficult to control the size of the cam surface.

(2) The profile of the cam surface that generates the specified relationship between an external force and displacement of the contact point between the two surfaces is calculated by solving the differential equation derived from the static equation between the two links. These force-displacement characteristics are useful to control the size of the cam surface, although this is not intuitive for designers.

(3) The proposed design methodology is valid because the measured force-displacement characteristics of some prototypes designed with the proposed methodology agreed very well with the theoretical characteristics.

(4) A planar closed-loop linkage with the MFCRPs can have both an adequate positioning accuracy to do the specified task and flexibility for safety in multiple directions. 
Since two links of the MFCRP are in sliding contact, some energy loss and wear between the two links occur. Therefore, it is necessary to further investigate about appropriate lubrication methods between the two links or appropriate materials for the two contact surfaces. These are future works of this research.

\section{Acknowledgements}

The authors are grateful to Stratasys Japan Co.Ltd. for helping to fabricate a prototype of the MFCRP with the material jetting. The authors are also grateful to DENSO Co.Ltd. for renting us the robot manipulator for this research. A part of this work was financially supported by JSPS KAKENHI Grant Number 18J21095.

\section{References}

Bidgory, H. J., Ahmadabadi, M. N. and Zakerzadeh, M. R., Design and Modeling of a Compact Rotational Nonlinear Spring, Proceedings of the 2016 IEEE International Conference on Intelligent Robots and Systems (IROS 2016), (2016), pp.4356-4361.

Hogan, N., Impedance Control: An Approach to Manipulation: Part 1-3, Journal of Dynamic Systems, Measurement, and Control, Vol.107 (1985), pp.1-24.

Jafali, A. et al., A New Actuator with Adjustable Stiffness Based on a Variable Ratio Lever Mechanism, IEEE/ASME Transaction on Mechatronics, Vol. 19, No. 1 (2014), pp.55-63.

Kashiri, N., Caldwell, D. G. and Tsagarakis, N., A Self-adaptive Variable Impedance Actuator Based on Intrinsic Nonlinear Compliance and Damping Principles, Proceedings of the 2017 IEEE International Conference on Robotics and Automation (ICRA 2017), (2017), pp.1248-1254.

Kim, B. and Deshpande, A. D., Design of Nonlinear Rotational Stiffness Using a Non-circular Pulley-spring Mechanism, Journal of Mechanisms and Robotics, Vol.6, No.4 (2014), 041109.

Kim, B.-S. and Song, J.-B., Hybrid Dual Actuator Unit: A Design of a Variable Stiffness Actuator based on an Adjustable Moment Arm Mechanism, Proceedings of the 2010 IEEE International conference on Robotics and Automation (ICRA 2010), (2010), pp. 1655-1660.

Kimura, N. Iwatsuki, N. and Ikeda, I., Design of a Flexibly-Constrained Revolute Pair with Non-linear Stiffness for Safe Robot Mechanisms, Journal of Robotics and Mechatronics, Vol.31, No.1 (2019), pp.156-165.

Migliore, S. A., Brown, E. A. and DeWeerth, S.P., Biologically Inspired Joint Stiffness Control, Proceedings of the 2005 IEEE International Conference on Robotics and Automation (ICRA 2005), (2005), pp. 4508-4513.

Okada, M. and Kino, S., Torque Transmission Mechanism with Nonlinear Passive Stiffness using Mechanical Singularity, Proceedings of the 2008 IEEE International Conference on Robotics and Automation (ICRA 2008), (2008), pp.17351740.

Palli, G. et al., Design of a Variable Stiffness Actuator Based on Flexures, Journal of Mechanisms and Robotics, Vol.3, No.3 (2011), 034501.

Park, J.-J. and Song, J.-B., A Nonlinear Stiffness Safe Joint Mechanism Design for Human Robot Interaction, Journal of Mechanical Design, Vol.132, No.6 (2010), 061005.

Petit, F. et.al., Analysis and Synthesis of the Bidirectional Antagonistic Variable Stiffness Mechanism, IEEE/ASME Transaction on Mechatronics, Vol. 20, No.2 (2015), pp.684-695.

Salisbury, J. K., Active Stiffness Control of a Manipulator in Cartesian Coordinates, Proceedings of the 19th IEEE Conference on Decision and Control, Vol. 19 (1980), pp.95-100.

Schepelmann, A., Geberth, K. A. and Geyer, H., Compact Nonlinear Springs with User Defined Torque-Deflection Profiles for Series Elastic Actuators, Proceedings of the 2014 IEEE International Conference on Robotics and Automation (ICRA 2014), (2014), pp.3411-3416.

Wolf, S. and Hirzinger, G., A New Variable Stiffness Design: Matching Requirements of the Next Robot Generation, Proceedings of the 2008 IEEE International Conference on Robotics and Automation (ICRA 2008), (2008), pp. 1741-1746.

Wolf, S., Eiberger, O. and Hirzinger, G., The DLR FSJ: Energy based design of a variable stiffness joint, Proceedings of the 2011 IEEE International Conference on Robotics and Automation (ICRA 2011), (2011), pp. 5082-5089. 